\title{
Avifauna do Parque Estadual de Vila Rica do Espírito Santo, Fênix, Paraná
}

\author{
Pedro Scherer Neto ${ }^{1,3}$ \& Arthur Angelo Bispo ${ }^{2}$ \\ ${ }^{1}$ Museu de História Natural “Capão da Imbuia”, Rua Benedito Conceição, 407, \\ CEP 82810-080, Curitiba, PR, Brasil \\ ${ }^{2}$ Laboratório de Ecologia Teórica e Síntese, Universidade Federal de Goiás - UFG, Goiânia, GO, Brasil \\ ${ }^{3}$ Autor para correspondência: Pedro Scherer Neto, e-mail: pedroschererneto@yahoo.com.br
}

SCHERER NETO, P. \& BISPO, A.A. Birds species composition of the Vila Rica do Espírito Santo State Park, Fênix, Paraná. Biota Neotrop. 11(3): http://www.biotaneotropica.org.br/v11n3/en/abstract?article+bn02311032011

\begin{abstract}
The avifauna of Vila Rica do Espírito Santo State Park (PV) was studied from 1982 until 2007 in order to obtain the bird species composition. This protected area is located in in Fênix, a Parana state municipality that is inserted in a region whose landscape is a mosaic of patches of semideciduous forest and extensive areas of grain crops, sugar cane and pasture. It has an area of 354 ha covered mostly by second growth with mature characteristics and some places with primary sucession. The inventory was conducted using the methods of visual and aural recognition of bird species together captures with mist nets, during four periods. First, in 1982 with one expedition, the second period with 40 field expeditions between 1986 and 1999 and the third and fourth periods conducted respectively, from 2002 to 2003 and 2006, totaling in more 16 expeditions. This research has results in the record of 259 species of birds belonging to 55 families and 22 orders. The predominance of species of the Passeriformes order and Suboscines suborder suggests that the bird assemblage of the PV is characterized by forest elements. This environment is the most representative in the study area and has the largest number of species, 189 species, which are branches species (164 spp.), trunk species (14 spp.) and ground species (11 spp.). The PV has 14 endangered species and high species richness and this way present an important role on the regional landscape as a protected area. This landscape composed by some patches is fundamental to maintenance of local diversity, especially when these forest are protected in some way, as the case Vila Rica do Espírito Santo State Park which is a protected area with restricted use.
\end{abstract}

Keywords: bird assemblages, inventories, Atlantic forest, conservation.

SCHERER NETO, P. \& BISPO, A.A. Avifauna do Parque Estadual de Vila Rica do Espírito Santo, Fênix, Paraná. Biota Neotrop. 11(3): http://www.biotaneotropica.org.br/v11n3/pt/abstract?article+bn02311032011

Resumo: A avifauna do Parque Estadual de Vila Rica do Espírito Santo foi estudada a partir de 1982 até o ano de 2007 com a finalidade de obter a sua composição de espécies de aves. Situado no município de Fênix, no estado do Paraná, essa unidade de conservação está inserida em uma região cuja paisagem é um mosaico de fragmentos da floresta Estacional Semidecidual entre extensas áreas destinadas ao plantio de grãos, cana-de-açúcar e pastagens. Possui uma área de 354 ha recoberta em sua maioria por uma floresta secundária em estádio avançado de regeneração e por capoeiras. O inventário foi realizado utilizando em conjunto os métodos de reconhecimento visual e auditivo e por capturas com redes ornitológicas, durante quatro períodos, uma primeira amostragem em 1982, o segundo período com 40 expedições a campo entre 1986 e 1999 e o terceiro e quarto períodos conduzidos, respectivamente, de 2002 a 2003 e 2006, totalizando em mais 16 expedições. Esta pesquisa revelou a ocorrência de 259 espécies de aves distribuídas em 55 famílias e 22 ordens. A predominância de espécies representantes da ordem Passeriformes e da subordem dos Suboscines sugere que a assembléia de aves do parque é caracterizada por elementos silvícolas. Esse ambiente é o mais representativo no parque e abriga o maior numero de espécies, 189 espécies, destacando-se a ocupação por aves tamnícolas (164 spp.), corticícolas (14 spp.) e terrícolas (11 spp). O PV por abrigar 14 espécies ameaçadas de extinção e uma alta riqueza de espécies apresenta uma alta importância na paisagem regional como uma unidade de conservação. Essa paisagem composta por alguns remanescentes é fundamental para a manutenção da diversidade local, ainda mais quando essas florestas estão protegidas de alguma forma, como o caso do Parque Estadual Vila Rica do Espírito Santo que é uma unidade de conservação de uso restrito.

Palavras-chave: assembléia de aves, inventário, floresta Atlântica, conservação. 


\section{Introdução}

O estado do Paraná possui uma combinação dos biomas Floresta Atlântica, Cerrado e Campos que possibilita uma elevada riqueza de espécies de aves no estado (660 espécies) (Scherer-Neto \& Straube 1995, Straube \& Urben-Filho 2001). Dos biomas encontradas no estado, a Floresta Atlântica é o mais representativo com uma cobertura vegetal natural que cobria cerca de $85 \%$ do território do estado e que com o passar dos anos, tornou-se um mosaico de pequenos conjuntos de remanescentes florestais, intercalados com grandes áreas modificadas pelo homem, representadas por monoculturas e demais atividades agro-pastoris (Pichorim \& Boçon 1996, Anjos 1998, Bornschein \& Reinert 2000). A formação mais afetada deste bioma pelo expansionismo agrícola, a Floresta Estacional Semidecidual, ficou reduzida a pequenos e esparsos fragmentos, distribuídos ao longo da sua área de distribuição (Maack 1981), restando, hoje menos de 5\% da área total original (Anjos 1998, Mikich \& Silva 2001). Essa destruição do habitat é uma das maiores ameaças à fauna do estado do Paraná (vide Mikich \& Bérnils 2004), sendo assim o estabelecimento de areas protegidas nos domínios desse tipo florestal é uma ferramenta efetiva para a conservação de sua fauna (Goerck 2006).

O noroeste do estado, composto principalmente pela Floresta Estacional Semidecidual, é uma das áreas consideradas prioritárias que necessitam de pesquisas ornitológicas sistemáticas no estado (Straube \& Urben-Filho 2001). Poucos foram os estudos realizados nesta região, mesmo em unidades de conservação tal como o Parque Estadual de Vila Rica do Espírito Santo. Nesse contexto, esse estudo teve como objetivo inventariar a assembléia de aves dessa unidade de conservação durante um longo período de amostragem e caracterizar sua composição específica de acordo com a sua frequiência de ocorrência, distribuição no ambiente, em relação aos hábitos migratórios e a presença nas categorias de espécies ameaçadas de extinção.

\section{Material e Métodos}

\section{1. Área de estudo}

A região de estudo localiza-se no município de Fênix, situado no terceiro planalto paranaense (Figura 1). O relevo é suavemente ondulado e a altitude média é de 650 m (ITCF 1987). O clima da região é subtropical úmido mesotérmico ou $\mathrm{C} f a$ (Köppen), com temperatura média dos meses mais quentes superior a $22{ }^{\circ} \mathrm{C}$ e dos meses mais frios, inferior a $18^{\circ} \mathrm{C}$. Os verões são quentes e as geadas pouco freqüentes, com tendência de concentração de chuvas entre os meses de dezembro e fevereiro, sem estação seca definida (ITCF 1978, Maack 1981).

O Parque Estadual Vila Rica do Espírito Santo (PV), com 354 ha, está localizado nas confluências dos rios Ivaí e Corumbataí ( $23^{\circ} 55^{\prime} \mathrm{S}$ e $51^{\circ} 57^{\prime} \mathrm{O}$ ) (Figura 1). O PV apresenta formato aproximadamente regular e em sua maior parte $(75 \%)$ está recoberto por floresta secundária em estádio avançado de regeneração, que resultou do abandono da cidade colonial espanhola de Villa Rica Del Espiritu Santo no ano de 1632 (Mikich \& Silva 2001). Além do ambiente floretal em diferentes estágios de regeneração em seu interior ainda pode ser encontrado um lago artificial e este remanescente está inserido em uma matriz composta por áreas cultivadas, pricipalmente com milho e soja, por pastagens entre outros remanescentes florestais (Mikich \& Silva 2001) (Figura 1).

\section{Coleta dos dados}

Os estudos com o objetivo de conhecer a avifauna no PV iniciaramse em 1982 resultando na primeira listagem de espécies do parque. Os registros foram feitos com base em métodos tradicionais da pesquisa ornitológica, ou seja, identificação das aves por reconhecimento visual e/ou auditivo durante caminhadas por estradas e trilhas existentes neste

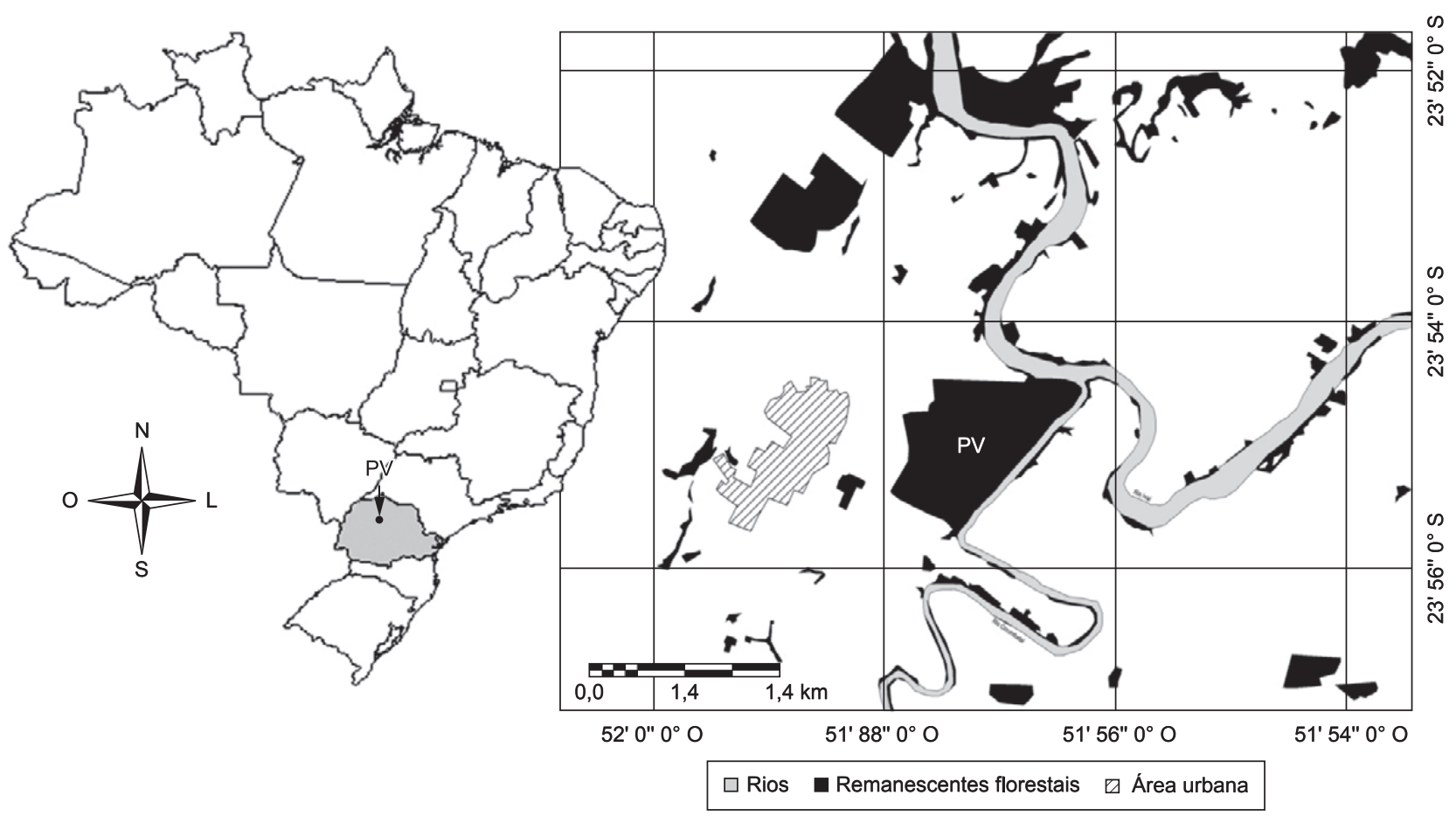

Figura 1. Localização do município de Fênix no estado do Paraná (esquerda) e o Parque Estadual Vila Rica do Espírito Santo (PV) e sua paisagem de entorno próxima a área urbana do município de Fênix.

Figure 1. Fênix municipality in the Parana state (left) and the Vila Rica do Espírito Santo State Park (PV) and the surroundings landscape nearest the urban area. 
parque ou por navegação nos rios Ivaí e Corumbataí. As observações foram conduzidas principalmente durante o dia, embora amostragens noturnas tenham sido eventualmente realizadas.

Entre 1986 e 1999 foram realizadas 40 expedições a esta unidade de conservação, com duração de até quatro dias, representando o maior esforço das investigações ornitológicas do PV. Em agosto de 1991, além dos registros visuais e auditivos, teve início um programa de captura e recaptura de aves com auxílio de redes ornitológicas, incluindo a marcação de indivíduos com anilhas metálicas alfa numérica fornecidas pelo Centro Nacional de Pesquisa para Conservação das Aves Silvestres (CEMAVE). Com isso, aumentou a probabilidade de registro de algumas espécies crípticas.

$\mathrm{O}$ terceiro e o quarto período de estudos avifaunísticos foram conduzidos, respectivamente, de 2002 a 2003 (12 campanhas) e em 2006 (quatro campanhas). Nessas etapas, as amostragens foram mais pontuais, realizadas em três parcelas de 1 ha, que foram selecionadas no interior do parque, de modo a inventariar a avifauna das subformações aluvial e submontana. Nessas parcelas, foram realizados registros visuais e auditivos, além de capturas com redes ornitológicas, durante, pelo menos, dois dias/mês.

Independente do período de estudo, a cada amostragem foi gerada uma lista das espécies registradas, permitindo a elaboração de uma listagem geral, além de análises sazonais, por ambiente, bem como a curva de acumulação de espécies (Mao Tau) e a curva de riqueza estimada (Chao 1) (Santos 2004). A frequiência de ocorrência das espécies foi calculada em relação às 57 amostragens, por meio da fórmula $F O=$ f/F. 100, no qual fé o número de fases em que a espécie foi registrada e F é o número total de fases de campo. De acordo com este índice, as espécies foram inseridas em categorias de frequiência, sendo estas: 1) alta, para espécies registradas em mais de $61 \%$ das amostragens ( $\mathrm{n} \geq 35$ fases); 2 ) média, para espécies registradas entre 31 a $60 \%$ das amostragens ( $34 \leq n \geq 18$ ), e 3 ) baixa, para espécies registradas abaixo de $30 \%$ das amostragens ( $\mathrm{n} \leq 17$ fases).

A ocupação de ambientes foi baseada na categorização das espécies por Straube (1995), que as divide em terrestres, aquáticas e aerícolas. As terrestres, por sua vez, foram subdivididas em campícolas (habitam áreas abertas), silvícolas (habitam as áreas florestais abrangendo as terrícolas (solo), tamnícolas (ramagem em qualquer estrato florestal) e corticícolas (troncos e cascas de árvores). Já as espécies aquáticas foram subdivididas em natantes (ocupam a lâmina d'água, promovendo natação e/ou mergulhos para a obtenção de alimento) e limícolas (vivem nas margens de corpos d'água com ou sem vegetação circundante). As espécies que usam o espaço aéreo na maior parte do ciclo circadiano foram consideradas aerícolas.

\section{Resultados e Discussão}

O total de 259 espécies de aves pertencentes a 55 famílias e 22 ordens foram identificadas no PV (Apêndice 1). Esse valor representa $39 \%$ do total de aves encontradas no estado do Paraná segundo Scherer-Neto \& Straube (1995) e, portanto, revela a importância desta área protegida para a conservação da avifauna paranaense. As espécies registradas no PV dividem-se em 113 (43,6\%) nãopasseriformes e $146(56,4 \%)$ Passeriformes, o que resulta em uma relação de 0,77:1 $(\mathrm{NP} / \mathrm{P}=0,773)$. Em relação às subordens de Passeriformes, foram identificadas 56 espécies $(21,6 \%$ da avifauna total) para o subgrupo dos Oscines e 90 espécies (34,7 \% da avifauna total) para o subgrupo dos Sub-Oscines. A relação entre estes grupos foi de 1,6:1 $(\mathrm{SO} / \mathrm{O}=1,607)$. Baseado nas características dessas ordens e subordens verifica-se que a assembléia de aves do PV é caracterizada principalmente por elementos silvícolas (cf Slud 1976, Willis 1976, Straube 1995, Pichorim \& Bóçon 1996, Sick 1997), pois a ordem Passeriformes possui um grande número de espécies com preferência por ambientes florestais, o que deve explicar a sua predominância no PV, enquanto que os não Passeriformes, por serem representados principalmente por espécies de maior porte, geralmente estão distribuídos em ambientes abertos, pouco comuns no interior do parque. Já a subordem Oscines apresenta espécies mais comuns às áreas abertas e bordas, enquanto que os Suboscines representam espécies mais intimamente relacionadas aos ambientes florestais (Haffer 1995 apud Anjos 2001).

A curva de acumulação de espécies gerada não estabilizou ao final de todas as amostragens apesar de ter ocorrido poucos acréscimos nas últimas expedições (Figura 2). A curva de riqueza estimada (Figura 2) sugere que alguns novos registros poderiam ocorrer para o PV. Esta diferença entre a riqueza estimada e a observada foi de 23 espécies o que representa apenas $9 \%$ da riqueza total observada. O número de espécies variou conforme o mês de amostragem (Tabela 1) e as flutuações observadas na composição específica no decorrer dos ciclos sazonais são comumente encontradas em estudos ornitológicos (vide Anjos \& Graf 1993, Marterer 1996, Abe 1997, Gimenes \& Anjos 2000, Bispo \& Scherer-Neto 2010), estando às épocas com maiores riquezas relacionadas com a presença de espécies migratórias e com o período reprodutivo das aves durante a primavera e o verão (Anjos \& Graf 1993, Gimenes \& Anjos 2000).

A análise da frequiência revelou que $66,8 \%$ das espécies possuem uma ocorrência baixa $(n=173), 21 \%$ média $(n=54)$ e $12,2 \%$ alta $(\mathrm{n}=32)$. A irregularidade nos registros de muitas espécies pode indicar uma tendência, mas não afirmar que a espécie desapareceu de um determinado local. A configuração ambiental do PV pode fornecer recursos que favorecem a existência e permanência de espécies em sua área, sendo que de acordo com a exigência ecológica de cada espécie essas podem aumentar sua ocorrência localmente. Cada grupo de espécies responde diferentemente aos processos que determinam sua ocorrência no parque. Desta maneira, a baixa frequiência de determinadas espécies pode seguir diferentes fatores.

Como exemplo, foi verificado que muitas espécies, principalmente não-passeriformes, ocorrem ocasionalmente no parque, como o urubu-rei Sarcoramphus papa, e alguns falconiformes em geral são avistados em vôo e muito raramente pousados. Por sua vez, algumas espécies de Passeriformes pertencentes às famílias Thamnophilidae, Grallaridae e Formicarridae, declinaram seus registros ao longo dos anos, pois apesar do esforço amostral ter sido diferenciado entre os anos de amostragem os métodos sempre focaram as espécies florestais o que pode comprovar o declínio principalmente para as espécies Hypoedaleus guttatus e Dysithamnus mentalis, os quais são representantes das famílias mencionadas. Outras espécies, tais como Mackenziaena leachii, M. severa, Pyriglena leucoptera, Chamaeza campanisona e Grallaria varia, apresentaram declínio no número de registros até o ponto de não serem mais registrados na última década. As três últimas espécies por sua vez ainda são encontradas na paisagem do entorno em um remanescente florestal de maior porte, a fazenda Barbacena, com 524 ha, localizada na margem direita do rio Ivaí. Estas famílias representadas por insetívoros que ocupam os estratos inferiores do interior florestal para forrageamento (Pearman 2002) e que tendem a permanecer em áreas de dossel fechado (Yabe \& Marques 2001) são mais sensíveis aos processos de fragmentação florestal (Willis 1979, Aleixo \& Vielliard 1995). Desta forma ocorre o aumento das chances de extinção destas espécies em remanescentes de menor porte e/ou com a perda da qualidade do seu microhabitat (p.ex. sub-bosque). Entre os piprídeos, algumas espécies se mantiveram constantes, como Pipra fasciicauda cuja ocorrência foi principalmente registrada por meio de capturas. Outras espécies desta família apresentaram baixas freqüências de ocorrência, tais como o tangará Chiroxiphia caudata o qual esteve ausente por longos períodos de amostragem, não registrada desde 1999. 
Scherer Neto, P. \& Bispo, A.A.

Tabela 1. Número de amostragens (NE) por ano no Parque Estadual de Vila Rica e as respectivas riquezas e a riqueza média por ano (M).

Table 1. Number of field expeditions (NE) per year in the Vila Rica State Park and the respective species richness and average (M) species richness per year.

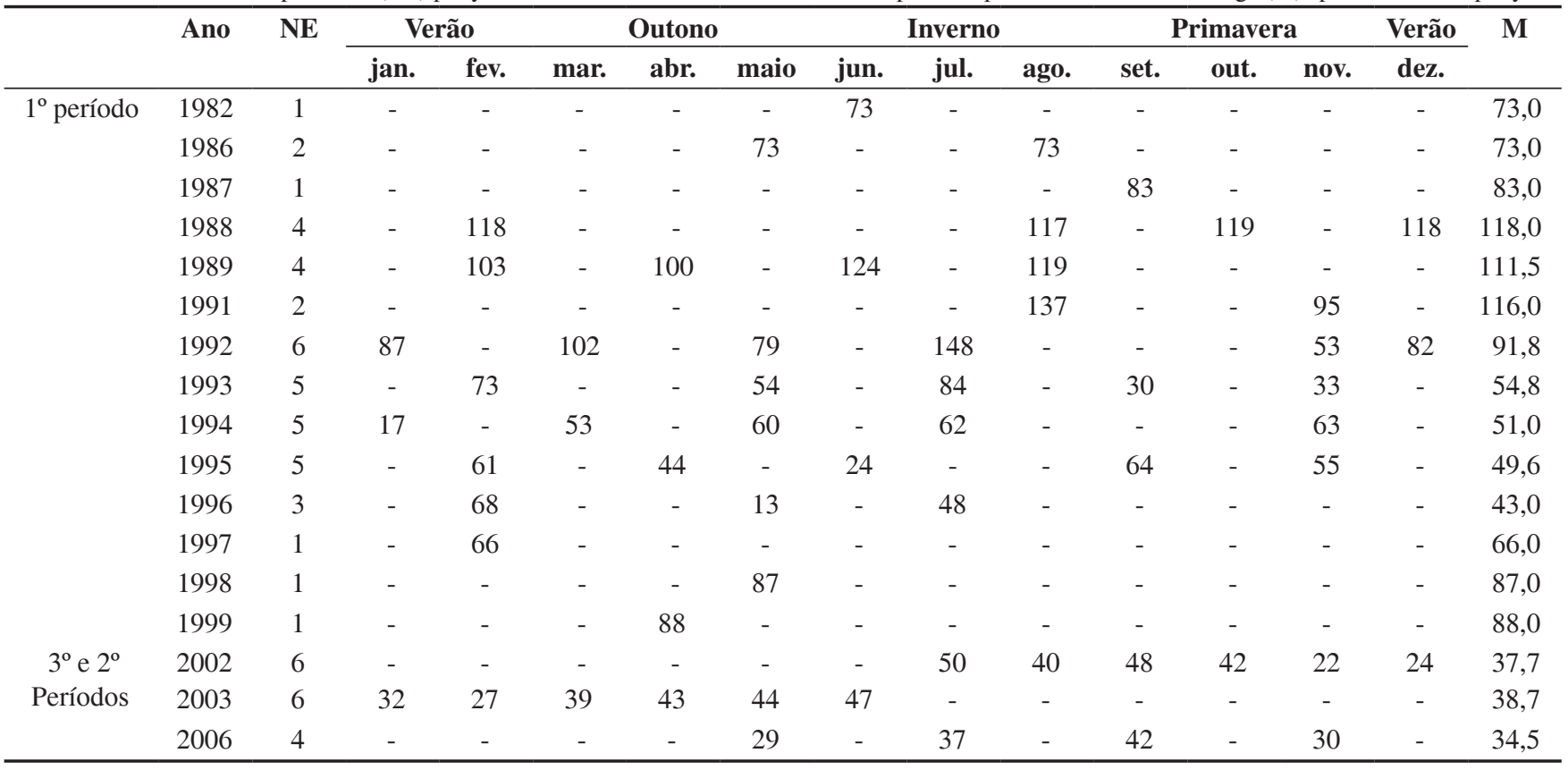

Tabela 2. Distribuição numérica da avifauna do PV nos tipos ecológicos sugeridos por Straube (1995).

Table 2. Numbers of species per ecologicals categories according Straube (1995).

\begin{tabular}{lccc}
\hline & Tipos ecológicos & $\mathbf{N}^{\mathbf{0}}$ de espécies \\
\hline Aquáticas & Natantes & & 05 \\
& Limícolas & & 21 \\
Terrestres & Silvícolas & Terrícolas & 11 \\
& & Tamnícolas & 164 \\
& & Corticícolas & 14 \\
& & & 35 \\
Aerícolas & Campícolas & & 09 \\
\hline
\end{tabular}

A plasticidade das aves na ocupação de ambientes varia entre as espécies, que podem ser restritas ou não a um único tipo ambiental. Sendo assim, ao existir a restrição de algumas espécies a tipos específicos de habitats, o número delas em um remanescente é provavelmente determinado pelo número de ambientes que ele contém (Simberloff \& Abele 1982, McIntyre 1995, Paglia et al. 1995). Assim, para uma melhor avaliação da avifauna dentro do $\mathrm{PV}$, há que considerar os diferentes ambientes que este contém e como alguns se desenvolveram ao longo do tempo. No início da década de 1980, quando foram iniciadas as pesquisas ornitológicas no PV, uma parte de sua área era destinada a um viveiro de árvores nativas e exóticas. Posteriormente, por indicação do Plano de Manejo (ITCF 1987), foi abandonado o cultivo de arbóreas e a área em questão evoluiu gradativamente para uma floresta secundária, circundada pela floresta em estádio avançado que recobre o restante do parque, embora continuasse a abrigar diversas espécies cultivadas. Alguns trechos do parque foram explorados no passado para diversos fins e não mostraram uma regeneração expressiva, provavelmente devido às características do solo. $\mathrm{O}$ ambiente silvícola, o mais representativo do
PV, é o que abriga maior número de espécies ( $\mathrm{n}=189,72 \%$ de toda avifauna), destacando-se a ocupação por aves tamnícolas (87\% dentre as silvícolas), corticícolas (7,3\%) e terrícolas (5,7\%) (Tabela 2). A presença de ambientes florestais em diversos estádios sucessionais no interior do parque permite, ainda, a ocupação de 35 espécies campícolas (Tabela 2). No caso específico da família Picidae, das nove espécies registradas nesse estudo, uma ocupa o ambiente campícola e as demais são corticícolas, ocupando os diversos microambientes no interior florestal. Nos ambientes aquáticos foram registradas 26 espécies e a ocorrência destas variou conforme o grupo analisado. $\mathrm{O}$ lago apresentou, ao longo do tempo, uma rica fauna de aves limícolas e natantes, com a presença constante de saracuras, frangos-d'água e jaçanãs, em oposição aos martim-pescadores, que não foram registrados na maioria das amostragens. Algumas espécies, como o biguá e biguatinga, não apresentaram uma alta ocorrência na área de estudo, como poderia se esperar em função da presença de grandes rios e do lago no interior, tendo sido registradas em apenas cinco amostragens. Entre as garças e socós, somente o socó-dorminhoco Nycticorax nycticorax e o socozinho Butorides striata utilizam regularmente o lago e as árvores localizadas nas margens deste. Entre os anatídeos registrados como natantes, somente o pato-selvagem Cairina moschata e a marreca-ananaí Amazonetta brasiliensis foram avistados no lago nos oito primeiros anos de pesquisa no parque. O irerê Dendrocygna viduata foi percebido na área de estudo deslocando-se durante seus vôos noturnos, e ocasionalmente foram visualizados em banhados e cultivos de arroz do entorno. A sucessão natural de alguns desses ambientes da área de estudo resultou em uma alteração de sua composição específica. Este fato não deve ser considerado prejudicial já que grande parte das espécies que se tornaram ausentes não são dependentes de ambientes florestais.

A maior parte $(n=247)$ das espécies registradas no parque é considerada residente. Foram registradas 12 espécies que realizam migrações regionais (residentes migratórios), sendo elas: os gaviões Elanoides forficatus e Ictinia plumbea, o andorinhão-do-temporal Chaetura meridionalis, os tiranídeos Pyrocephalus rubinus, 


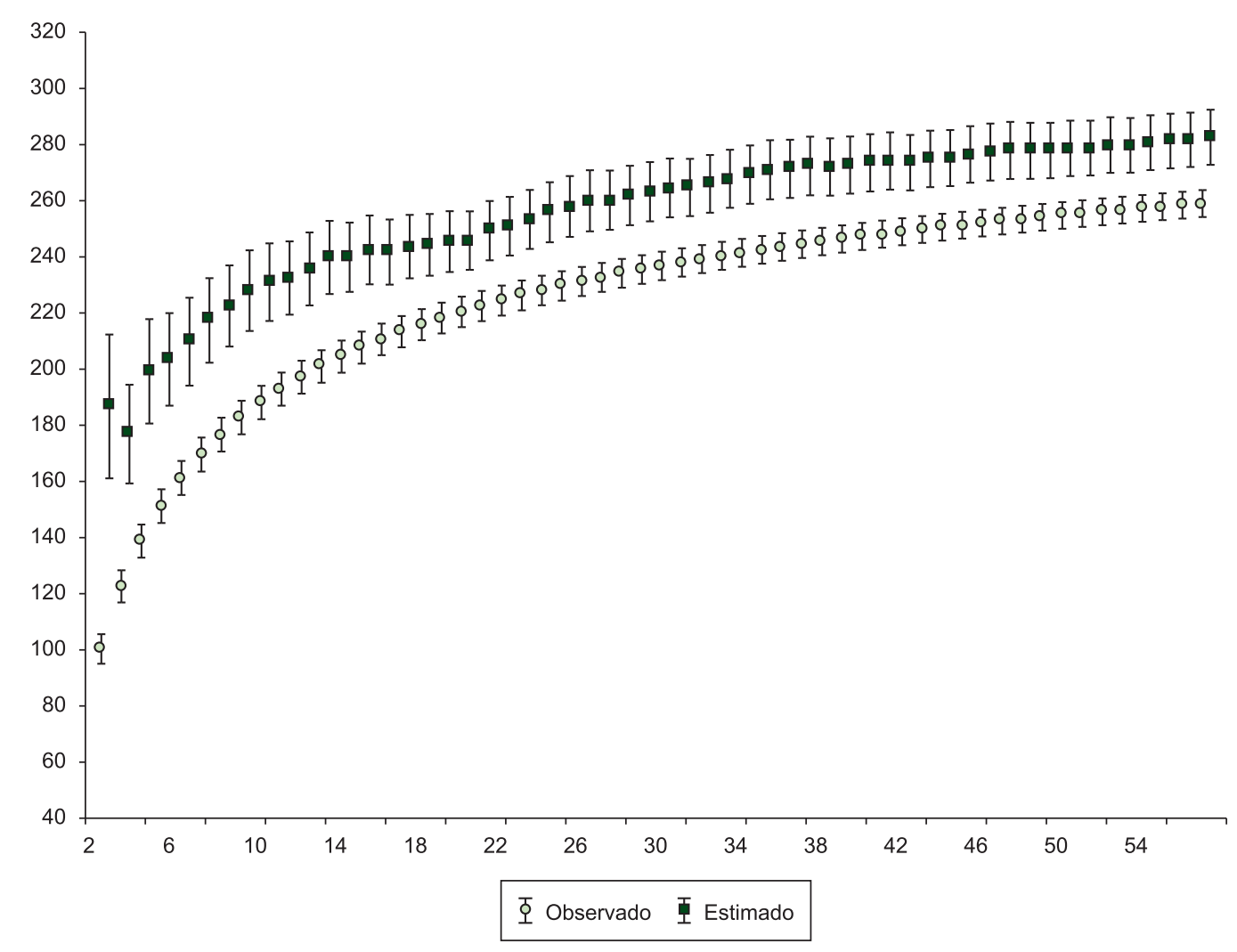

Figura 2. Curva acumulada de espécies (Mao Tau) ao longo das 57 amostragens realizadas no Parque Estadual Vila Rica do Espírito Santo, durante o período de 1982 a 2006 e a curva estimada de espécies (Chao 1) baseada nessas amostragens.

Figure 2. Accumulated species curve (Mao Tau) showing the increase of species richness during the 57 field expeditions in the Vila Rica do Espírito Santo State Park, during the years 1982 until 2006 and the estimated species richness curve (Chao 1) based in this samples.

Tyrannus melancholicus e Tyrannus savana, as andorinhas Progne tapera, Progne chalybea, Pygochelidon cyanoleuca e Stelgidopteryx ruficollis e os sabiás Turdus amaurochalinus e Turdus subalaris. Quanto aos migrantes de longa distância (visitantes do hemisfério norte) foi registrada a andorinha-de-bando Hirundo rustica. Em consequiência de seus hábitos migratórios e seus registros esporádicos, dez espécies mencionadas para o PV enquadram-se na categoria de baixa frequiência de ocorrência, e outras duas enquadradas na categoria de média frequiência (Apêndice 1). Ao longo dos anos, as fases de campo não foram regulares em todos os meses ou nos mesmos meses. Todavia cada estação foi amostrada pelo menos uma vez no ano tendo sido possível estimar um padrão de ocorrência das espécies migratórias (latu sensu). O sovi Ictinia plumbea foi registrado em 16 amostragens, ocorrendo no início da primavera e verão, sendo seus registros concentrados nos meses de agosto a fevereiro. No ano de 2002, quando as expedições foram mensais, a espécie foi registrada de setembro a janeiro. O andorinhão-do-temporal Chaetura meridionalis apareceu em 12 amostragens, concentradas entre fevereiro de 1988 e julho de 1992, não sendo verificado um padrão sazonal de distribuição. A tesourinha Tyrannus savana foi registrada principalmente no final da primavera e verão, apesar de ter sido eventualmente encontrada fora desse período, como junho de 1982 e agosto de 1989. Dentre as andorinhas, a andorinhadoméstica-grande Progne chalybea, aparentemente, foi a que apresentou maior sazonalidade nos registros, estando ausente nos meses de inverno. As demais espécies da família Hirundinidae foram registradas em todas as estações, apesar de ocorrer um maior número de registros nos meses de julho e agosto (inverno). O sabiá-ferreiro Turdus subalaris foi registrado no PV apenas nas estações de inverno e primavera, ao contrário do sabiá-poca Turdus amaurochalinus, com registros em 58\% das amostragens ao longo de todo o ciclo sazonal, demonstrando ser uma espécie residente localmente. As demais espécies consideradas migratórias por terem sido registradas apenas uma vez impossibilitaram determinar o padrão de ocorrência destas na área de estudo.

Foram registradas 14 espécies de aves incluídas em diferentes categorias de ameaça na literatura especializada. Dentre estas, oito foram incluídas pela BirdLife (2000) no "Threatened Birds of the World", sendo sete na categoria "quase ameaçada" (near-threatened) e uma na categoria de vulnerável. As demais espécies são mencionadas no "Livro Vermelho da Fauna Ameaçada no Estado do Paraná" (Mikich \& Bérnils 2004), sendo cinco na categoria de "dados deficientes", uma na categoria "vulnerável" e uma na categoria de "quase ameaçada" (Apêndice 1). Dessas espécies apenas uma foi mencionada nas duas listas, o chibante Laniisona elegans. Entre as espécies ameaçadas registradas para o PV, 12 foram categorizadas com baixa freqüência de ocorrência, sendo que nenhuma delas foi registrada pelos autores após 1999. O azulinho Cyanoloxia glaucocaerulea e o araçari-banana, Pteroglossus bailoni foram registrados apenas em fragmentos florestais do entorno após este período. O araçari-de-bico-branco Pteroglossus aracari, vulnerável na lista estadual (Mikich \& Bérnils 2004), apareceu em quase $70 \%$ das amostragens na primeira etapa de pesquisa. Também apareceu em $60 \%$ das amostragens em 2002 e em $40 \%$ de 2005, sempre em bandos de até sete indivíduos. 


\section{Agradecimentos}

Os autores agradecem outros profissionais e auxiliares de campo que contribuíram para o estudo da avifauna no Parque Estadual de Vila Rica do Espírito Santo: Fernando C. Straube, Alberto Urben Filho, Douglas Kajiwara, Luiz dos Anjos, Eduardo Carrano, Cassiano Fadel Ribas, Valdi Paula Gonçalves, Luiz Carlos Sieben, Mauro M. Britto, Pedro Sanches Filho, Elizeu Souza Pinto, Luiz F. F. de Macedo, Sebastião Carlos Pereira, Valdenisio Ferreira dos Santos, Adão Schoroeder, Carlos Schicowski, Valter Nicolack, Pedro Senna Maia, Aline Dal'Maso Ferreira, Daniel Isolani, Mariana Sant'Ana Schlichting, Leonardo Giraldi Gustmann e Marcos Nakagawa. Agradecemos a Gledson Vigiano Bianconi, Sandra Bos Mikich e aos revisores da Biota Neotropica pelas sugestões ao manuscrito.

\section{Referências Bibliográficas}

ABE, LM. 1997. Estudo da avifauna em remanescentes florestais contíguos a reflorestamentos com Pinus elliottii Elgelm, 1880. Estud. Biol. 41:37-60.

ALEIXO, A. \& VIELLIARD, J.M.E. 1995. Composição e dinâmica da avifauna da mata de Santa Genebra, Campinas, São Paulo, Brasil. Rev. Bras. Zool. 12(3):493-511.

ANJOS, L. 1998. Conseqüências biológicas da fragmentação no norte do Paraná. IPEF, Sér. Técn. 12(32):87-94.

ANJOS, L. 2001. Comunidades de aves florestais: implicações na conservação. In Ornitologia e conservação: da ciência às estratégias (J.L.B. Albuquerque, J.F. Cândido Junior, F.C. Straube \& A.L. Roos, ed) Sociedade Brasileira de Ornitologia, Curitiba, p.17-37.

ANJOS L. \& GRAF, V. 1993. Riqueza de aves da Fazenda Santa Rita, região dos campos gerais, Palmeira, Paraná, Brasil. Rev. Bras. Zool. 10(4):673-693.

BIRDLIFE. 2000. Threatened birds of the world. Lynx Edicions and BirdLife International, Barcelona; Cambrigde.

BISPO, A.A. \& SCHERER-NETO, P. 2010. Taxocenose de aves em um remanescente da Floresta com Araucária no sudeste do Paraná, Brasil. Biota Neotrop.10(1): http://www.biotaneotropica.org.br/v10n1/pt/ abstract?article+bn02010012010

BORNSCHEIN, M.R. \& REINERT, B.L. 2000. Aves de três remanescentes florestais do norte do estado do Paraná, sul do Brasil, com sugestões para a conservação e manejo. Rev. Bras. Zool. 17(3):615-636. http://dx.doi. org/10.1590/S0101-81752000000300008

COMITÊ BRASILEIRO DE REGISTROS ORNITOLÓGICOS - CBRO. 2010. Lista das aves do Brasil. Versão out. 2010. CBRO. http://www. cbro.org.br.

GIMENES, M.R. \& ANJOS, L. 2000. Distribuição espacial de aves em um fragmento florestal do campus da Universidade Estadual de Londrina, norte do Paraná, Brasil. Rev. Bras. Zool. 17(1):263-271.

GOERCK, J.M. 2006. Conservação de Aves na Região do Domínio da Mata Atlântica no Brasil. In Áreas importantes para a conservação das aves no Brasil: parte 1 - estados do domínio da Mata Atlântica (G.A. Bencke, G.N. Maurício, P.F. Develey \& J.M. Goerck, orgs.). SAVE Brasil, São Paulo, p.17-24.

INSTITUTO AGRONÔMICO DO PARANÁ - IAPAR. 1978. Cartas climáticas do Estado do Paraná. Fundação Instituto Agronômico do Paraná, Londrina.
INSTITUTO DE TERRAS CARTOGRAFIA E FLORESTAS - ITCF. 1987. Plano de Manejo do Parque Estadual de Vila Rica do Espírito Santo, Fênix - PR. ITCF, Curitiba.

MAACK, R. 1981. Geografia física do Estado do Paraná. 2nd ed. J. Olympio, Rio de Janeiro.

MARTERER, B.T.P. 1996. Avifauna do Parque Botânico do Morro do Baú. FATMA, Florianópolis.

McINTYRE, N.E. 1995. Effects of Forest patch size on avian diversity. Landsc. Ecol. 10(2):85-99.

MIKICH, S.B. \& BÉRNILS, R.S. 2004. Livro Vermelho da Fauna Ameaçada no Estado do Paraná. Instituto Ambiental do Paraná, Curitiba. CD-ROM.

MIKICH, S.B. \& SILVA, S.M. 2001. Composição florística e fenologia das espécies zoocóricas de remanescentes de Floresta Estacional Semidecidual no centro-oeste do Paraná, Brasil. Acta Bot. Bras. 15(1):89-113.

PAGLIA, A.P., MARCO JUNIOR, P., COSTA, F.M., PEREIRA, R.F. \& LESSA, G. 1995. Heterogeneidade estrutural e diversidade de pequenos mamíferos em um fragmento de mata secundária de Minas Gerais, Brasil. Rev. Bras. Zool. 12(1):67-79. http://dx.doi.org/10.1590/S010181751995000100010

PEARMAN, P.B. 2002. The scale of community structure: habitat variation and avian guilds in tropical forest undestory. Ecol. Monogr. 72(1):19-39. http://dx.doi.org/10.1890/0012-9615(2002)072[0019:TSOCSH]2.0.CO;2

PICHORIM, M. \& BÓÇON, R. 1996. Estudo da composição avifaunística dos municípios de Rio Azul e Mallet, Paraná, Brasil. Acta Biol. Leopoldensia 18(1):129-144.

SANTOS, A.J. 2004. Estimativas de riqueza em espécies. In Métodos de estudos em Biologia da Conservação e Manejo da Vida Silvestre (L. Cullen Junior, C. Valladares-Padua e R. Rudran, orgs.). Ed. da UFPR; Fundação O Boticário de Proteção à Natureza, Curitiba.

SCHERER-NETO, P. \& STRAUBE, F.C. 1995. Aves do Paraná: história, lista anotada e bibliografia. Logo Press, Curitiba, 79p.

SICK, H. 1997. Ornitologia Brasileira. Nova Fronteira, Rio de Janeiro, 912p.

SIMBERLOFF, D. \& ABELE, L.G. 1982. Refuge design and island biogeographic theory: effects of fragmentation. Am. Naturalist 120(1):4150. http://dx.doi.org/10.1086/283968

SLUD, P. 1976. Geographic and climatic relationships of avifaunas with special reference to comparative distribuition in the Neotropics. Smithson. Contrib. Zool. 212:1-149. http://dx.doi.org/10.5479/si.00810282.212

STRAUBE, F.C. 1995. Métodos de caracterização e diagnóstico de avifaunas para estudos de impactos ambientais. In Manual de Avaliação de Impactos Ambientais - MAIA (P. Juchen, ed.). IAP/GTZ, Curitiba, Suplemento 2, p.1-15.

STRAUBE, F.C. \& URBEN-FILHO, A. 2001. Análise do conhecimento ornitológico da região noroeste do Paraná e áreas adjacentes, In Ornitologia e conservação: da ciência às estratégias (J.L.B. Albuquerque, J.F. Cândido Junior, F.C. Straube \& A.L. Roos, eds.) Sociedade Brasileira de Ornitologia, Curitiba, p.223-230.

WILLIS, E.O. 1976. Effects of a cold wave on an Amazonian avifauna in the upper Paraguay drainage, western mato grosso, and suggestions on oscine-suboscine relationships. Acta Amaz. 6(3):379-394.

WILLIS, E.O. 1979. The compositon on avian communities in remanescent woodlots in southern Brazil. Pap. Avul. Zool. 33(1):1-25.

YABE, R.S. \& MARQUES, E.J. 2001. Deslocamentos de aves entre capões no pantanal Mato-grossense e sua relação com a dieta. In Ornitologia e conservação: da ciência às estratégias (J.L.B. Albuquerque, J.F. Cândido Junior, F.C. Straube \& A.L. Roos, eds.) Sociedade Brasileira de Ornitologia, Curitiba, p.103-124. 
Apêndice 1. Lista de táxons registrados no Parque Estadual de Vila Rica do Espírito Santo entre 1982 a 2006, com espécies ameaçadas (EA) nas listas internacional (I) e estadual (E) (BirdLife 2000, Mikich \& Bérnils 2004).

Appendix 1. Taxons recorded in the Vila Rica do Espírito Santo State Park between 1982 and 2006, with endangered species (EA) in the international (I) and state lists (E) (BirdLife 2000, Mikich \& Bérnils 2004).

\begin{tabular}{|c|c|c|c|c|c|}
\hline Taxons & Nome popular & EA (I-E) & ST & FO $(\%)$ & DA \\
\hline \multicolumn{6}{|l|}{ Ordem Tinamiformes Huxley, 1872} \\
\hline \multicolumn{6}{|l|}{ Família Tinamidae Gray, 1840} \\
\hline Crypturellus obsoletus (Temminck, 1815) & inhambuguaçu & & $\mathrm{r}$ & 43,1 & te \\
\hline Crypturellus parvirostris (Wagler, 1827) & inhambu-chororó & & $\mathrm{r}$ & 28,07 & te \\
\hline Crypturellus tataupa (Temminck, 1815) & inhambu-chintã & & $\mathrm{r}$ & 57,89 & te \\
\hline Rhynchotus rufescens (Temminck, 1815) & perdiz & & $\mathrm{r}$ & 3,51 & camp \\
\hline \multicolumn{6}{|l|}{ Ordem Anseriformes Linnaeus, 1758} \\
\hline \multicolumn{6}{|l|}{ Família Anatidae Leach, 1820} \\
\hline Dendrocygna viduata (Linnaeus, 1766) & irerê & & $\mathrm{r}$ & 7,02 & nat \\
\hline Cairina moschata (Linnaeus, 1758) & pato-do-mato & & $\mathrm{r}$ & 15,79 & nat \\
\hline Amazonetta brasiliensis (Gmelin, 1789) & pé-vermelho & & $\mathrm{r}$ & 17,54 & nat \\
\hline \multicolumn{6}{|l|}{ Ordem Galliformes Linnaeus, 1758} \\
\hline Penelope superciliaris Temminck, 1815 & jacupemba & & r & 70,18 & ta \\
\hline \multicolumn{6}{|l|}{ Família Odontophoridae Gould, 1844} \\
\hline Odontophorus capueira (Spix, 1825) & uru & & $\mathrm{r}$ & 15,79 & te \\
\hline \multicolumn{6}{|l|}{ Ordem Podicipediformes Fürbringer, 1888} \\
\hline Podilymbus podiceps (Linnaeus, 1758) & mergulhão-caçador & & $\mathrm{r}$ & 1,75 & nat \\
\hline \multicolumn{6}{|l|}{ Ordem Suliformes Sharpe, 1891} \\
\hline \multicolumn{6}{|l|}{ Família Phalacrocoracidae Reichenbach, 1849} \\
\hline Phalacrocorax brasilianus (Gmelin, 1789) & biguá & & $\mathrm{r}$ & 8,77 & $\lim$ \\
\hline \multicolumn{6}{|l|}{ Família Anhingidae Reichenbach, 1849} \\
\hline Anhinga anhinga (Linnaeus, 1766) & biguatinga & & $\mathrm{r}$ & 10,53 & $\lim$ \\
\hline \multicolumn{6}{|l|}{ Ordem Pelecaniformes Sharpe 1891} \\
\hline Tigrisoma lineatum (Boddaert, 1783) & socó-boi & & $\mathrm{r}$ & 1,75 & $\lim$ \\
\hline Nycticorax nycticorax (Linnaeus, 1758) & savacu & & $\mathrm{r}$ & 47,37 & $\lim$ \\
\hline Butorides striata (Linnaeus, 1758) & socozinho & & $\mathrm{r}$ & 29,82 & $\lim$ \\
\hline Ardea cocoi Linnaeus, 1766 & garça-moura & & $\mathrm{r}$ & 8,77 & $\lim$ \\
\hline Ardea alba Linnaeus, 1758 & garça-branca-grande & & $\mathrm{r}$ & 3,51 & $\lim$ \\
\hline Egretta thula (Molina, 1782) & garça-branca-pequena & & $\mathrm{r}$ & 14,04 & $\lim$ \\
\hline \multicolumn{6}{|l|}{ Ordem Cathartiformes Seebohm, 1890} \\
\hline Cathartes aura (Linnaeus, 1758) & urubu-de-cabeça-vermelha & & $\mathrm{r}$ & 19,3 & aeri \\
\hline Coragyps atratus (Bechstein, 1793) & urubu-de-cabeça-preta & & $\mathrm{r}$ & 56,14 & aeri \\
\hline Sarcoramphus papa (Linnaeus, 1758) & urubu-rei & & $\mathrm{r}$ & 8,77 & aeri \\
\hline \multicolumn{6}{|l|}{ Ordem Accipitriformes Bonaparte, 1831} \\
\hline \multicolumn{6}{|l|}{ Família Accipitridae Vigors, 1824} \\
\hline Elanoides forficatus (Linnaeus, 1758) & gavião-tesoura & & $\mathrm{rm}$ & 1,75 & aeri \\
\hline Elanus leucurus (Vieillot, 1818) & gavião-peneira & & $\mathrm{r}$ & 3,51 & camp \\
\hline Harpagus diodon (Temminck, 1823) & gavião-bombachinha & & $\mathrm{r}$ & 3,51 & ta \\
\hline Ictinia plumbea (Gmelin, 1788) & sovi & & $\mathrm{rm}$ & 28,07 & ta \\
\hline Geranospiza caerulescens (Vieillot, 1817) & gavião-pernilongo & & $\mathrm{r}$ & 3,51 & ta \\
\hline Rupornis magnirostris (Gmelin, 1788) & gavião-carijó & & $\mathrm{r}$ & 66,67 & ta \\
\hline Parabuteo leucorrhous (Quoy \& Gaimard, 1824) & gavião-de-sobre-branco & $(\mathrm{E}-\mathrm{DD})$ & $\mathrm{r}$ & 7,02 & ta \\
\hline Geranoaetus melanoleucus (Vieillot, 1819) & águia-chilena & & $\mathrm{r}$ & 1,75 & aeri \\
\hline Buteo brachyurus Vieillot, 1816 & gavião-de-cauda-curta & & $\mathrm{r}$ & 8,77 & aeri \\
\hline
\end{tabular}

NT = quase ameaçadas; VU- vulnerável e DD = dados deficientes; Status (St), onde r = residente; rm = residente migratório; vn = visitantes do hemisfério norte. Freqüência de ocorrência $(\mathrm{FO} \%)$; Distribuição no ambiente $(\mathrm{DA})$, onde te = terrícola; ta = tamnícola; co = corticícola; nat = natante; lim = limícola; camp = campícola; aeri = aerícola. Ordenamento taxonômico segue CBRO (2010).

NT = almost endangered; VU- vulnerable e DD = insuficient data; Ocorrence frequency $($ FO $)$; Habitat distribution $($ DA $)$, being, te = ground species; ta = branches species; $c o=$ trunk species; nat = waterline species; lim = adjancent water areas; camp = prairie species; aeri $=$ aerial species. Taxonomic order recommended by CBRO (2010). 
Apêndice 1. Continuação...

Appendix 1. Continued..

\begin{tabular}{|c|c|c|c|c|c|}
\hline Taxons & Nome popular & EA (I-E) & ST & FO $(\%)$ & DA \\
\hline \multicolumn{6}{|l|}{ Ordem Falconiformes Bonaparte, 1831} \\
\hline \multicolumn{6}{|l|}{ Família Falconidae Leach, 1820} \\
\hline Caracara plancus (Miller, 1777) & caracará & & $\mathrm{r}$ & 36,84 & camp \\
\hline Milvago chimachima (Vieillot, 1816) & carrapateiro & & $\mathrm{r}$ & 21,05 & camp \\
\hline Herpetotheres cachinnans (Linnaeus, 1758) & acauã & & $\mathrm{r}$ & 7,02 & ta \\
\hline Micrastur semitorquatus (Vieillot, 1817) & falcão-relógio & & $\mathrm{r}$ & 5,26 & ta \\
\hline Falco sparverius Linnaeus, 1758 & quiriquiri & & $\mathrm{r}$ & 1,75 & camp \\
\hline \multicolumn{6}{|l|}{ Ordem Gruiformes Bonaparte, 1854} \\
\hline \multicolumn{6}{|l|}{ Família Aramidae Bonaparte, 1852} \\
\hline Aramus guarauna (Linnaeus, 1766) & carão & & $\mathrm{r}$ & 5,26 & $\lim$ \\
\hline \multicolumn{6}{|l|}{ Família Rallidae Rafinesque, 1815} \\
\hline Aramides cajanea (Statius Muller, 1776) & saracura-três-potes & & $\mathrm{r}$ & 19,3 & $\lim$ \\
\hline Aramides saracura (Spix, 1825) & saracura-do-mato & & $\mathrm{r}$ & 71,93 & $\lim$ \\
\hline Pardirallus nigricans (Vieillot, 1819) & saracura-sanã & & $\mathrm{r}$ & 35,09 & $\lim$ \\
\hline Pardirallus sanguinolentus (Swainson, 1837) & saracura-do-banhado & & $\mathrm{r}$ & 5,26 & $\lim$ \\
\hline Gallinula galeata (Lichtenstein, 1818) & frango-d'água-comum & & $\mathrm{r}$ & 38,6 & nat \\
\hline Porphyrio martinica (Linnaeus, 1766) & frango-d'água-azul & & $\mathrm{r}$ & 3,51 & $\lim$ \\
\hline \multicolumn{6}{|l|}{ Ordem Charadriiformes Huxley, 1867} \\
\hline \multicolumn{6}{|l|}{ Família Charadriidae Leach, 1820} \\
\hline Vanellus chilensis (Molina, 1782) & quero-quero & & $\mathrm{r}$ & 10,53 & camp \\
\hline \multicolumn{6}{|l|}{ Família Jacanidae Chenu \& Des Murs, 1854} \\
\hline Jacana jacana (Linnaeus, 1766) & jaçanã & & $\mathrm{r}$ & 49,12 & $\lim$ \\
\hline \multicolumn{6}{|l|}{ Ordem Columbiformes Latham, 1790} \\
\hline \multicolumn{6}{|l|}{ Família Columbidae Leach, 1820} \\
\hline Columbina talpacoti (Temminck, 1811) & rolinha-roxa & & $\mathrm{r}$ & 17,54 & ta \\
\hline Columbina picui (Temminck, 1813) & rolinha-picui & & $\mathrm{r}$ & 45,61 & camp \\
\hline Claravis pretiosa (Ferrari-Perez, 1886) & pararu-azul & & $\mathrm{r}$ & 7,02 & camp \\
\hline Patagioenas picazuro (Temminck, 1813) & pombão & & $\mathrm{r}$ & 87,72 & ta \\
\hline Patagioenas cayennensis (Bonnaterre, 1792) & pomba-galega & & $\mathrm{r}$ & 14,04 & ta \\
\hline Patagioenas plumbea (Vieillot, 1818) & pomba-amargosa & & $\mathrm{r}$ & 3,51 & ta \\
\hline Zenaida auriculata (Des Murs, 1847) & pomba-de-bando & & $\mathrm{r}$ & 50,88 & camp \\
\hline Leptotila verreauxi Bonaparte, 1855 & juriti-pupu & & $\mathrm{r}$ & 59,65 & ta \\
\hline Leptotila rufaxilla (Richard \& Bernard, 1792) & juriti-gemedeira & & $\mathrm{r}$ & 70,18 & ta \\
\hline Geotrygon montana (Linnaeus, 1758) & pariri & & $\mathrm{r}$ & 3,51 & ta \\
\hline \multicolumn{6}{|l|}{ Ordem Psittaciformes Wagler, 1830} \\
\hline \multicolumn{6}{|l|}{ Família Psittacidae Rafinesque, 1815} \\
\hline Aratinga leucophthalma (Statius Muller, 1776) & periquitão-maracanã & & $\mathrm{r}$ & 15,79 & ta \\
\hline Pyrrhura frontalis (Vieillot, 1817) & tiriba-de-testa-vermelha & & $\mathrm{r}$ & 84,21 & ta \\
\hline Forpus xanthopterygius (Spix, 1824) & tuim & & $\mathrm{r}$ & 3,51 & ta \\
\hline Brotogeris chiriri (Vieillot, 1818) & periquito-de-encontro-amarelo & & $\mathrm{r}$ & 7,02 & ta \\
\hline Pionopsitta pileata (Scopoli, 1769) & cuiú-cuiú & (I - NT) & $\mathrm{r}$ & 54,39 & ta \\
\hline Pionus maximiliani (Kuhl, 1820) & maitaca-verde & & $\mathrm{r}$ & 84,21 & ta \\
\hline \multicolumn{6}{|l|}{ Ordem Cuculiformes Wagler, 1830} \\
\hline \multicolumn{6}{|l|}{ Família Cuculidae Leach, 1820} \\
\hline Piaya cayana (Linnaeus, 1766) & alma-de-gato & & $\mathrm{r}$ & 84,21 & ta \\
\hline Coccyzus melacoryphus Vieillot, 1817 & papa-lagarta-acanelado & & $\mathrm{r}$ & 7,02 & ta \\
\hline Coccyzus euleri Cabanis, 1873 & papa-lagarta-de-euler & $(\mathrm{E}-\mathrm{DD})$ & $\mathrm{r}$ & 1,75 & ta \\
\hline Crotophaga major Gmelin, 1788 & anu-coroca & & $\mathrm{r}$ & 33,33 & $\lim$ \\
\hline
\end{tabular}

$\mathrm{NT}=$ quase ameaçadas; VU- vulnerável e DD = dados deficientes; Status (St), onde $\mathrm{r}=$ residente; $r \mathrm{rm}=$ residente migratório; vn = visitantes do hemisfério norte. Freqüência de ocorrência (FO\%); Distribuição no ambiente $(\mathrm{DA})$, onde te = terrícola; ta = tamnícola; co = corticícola; nat = natante; lim = limícola; camp = campícola; aeri $=$ aerícola. Ordenamento taxonômico segue CBRO (2010).

NT = almost endangered; VU- vulnerable e DD = insuficient data; Ocorrence frequency (FO); Habitat distribution (DA), being, te = ground species; ta = branches species; $c o=$ trunk species; nat $=$ waterline species; $\lim =$ adjancent water areas; camp $=$ prairie species; aeri $=$ aerial species. Taxonomic order recommended by CBRO (2010). 
Apêndice 1. Continuação...

Appendix 1. Continued...

\begin{tabular}{|c|c|c|c|c|c|}
\hline Taxons & Nome popular & EA (I-E) & ST & FO $(\%)$ & DA \\
\hline Crotophaga ani Linnaeus, 1758 & anu-preto & & $\mathrm{r}$ & 54,39 & camp \\
\hline Guira guira (Gmelin, 1788) & anu-branco & & $\mathrm{r}$ & 26,32 & camp \\
\hline Tapera naevia (Linnaeus, 1766) & saci & & $\mathrm{r}$ & 47,37 & ta \\
\hline Dromococcyx pavoninus Pelzeln, 1870 & peixe-frito-pavonino & & $\mathrm{r}$ & 3,51 & ta \\
\hline \multicolumn{6}{|l|}{ Ordem Strigiformes Wagler, 1830} \\
\hline \multicolumn{6}{|l|}{ Família Strigidae Leach, 1820} \\
\hline Megascops choliba (Vieillot, 1817) & corujinha-do-mato & & $\mathrm{r}$ & 33,33 & ta \\
\hline Strix hylophila Temminck, 1825 & coruja-listrada & & $\mathrm{r}$ & 17,54 & ta \\
\hline Strix huhula Daudin, 1800 & coruja-preta & $(\mathrm{E}-\mathrm{DD})$ & $\mathrm{r}$ & 3,51 & ta \\
\hline Glaucidium brasilianum (Gmelin, 1788) & caburé & & $\mathrm{r}$ & 61,4 & ta \\
\hline Athene cunicularia (Molina, 1782) & coruja-buraqueira & & $\mathrm{r}$ & 1,75 & camp \\
\hline Asio clamator (Vieillot, 1808) & coruja-orelhuda & & $\mathrm{r}$ & 3,51 & ta \\
\hline \multicolumn{6}{|l|}{ Ordem Caprimulgiformes Ridgway, 1881} \\
\hline Nyctibius griseus (Gmelin, 1789) & mãe-da-lua & & $\mathrm{r}$ & 3,51 & camp \\
\hline \multicolumn{6}{|l|}{ Família Caprimulgidae Vigors, 1825} \\
\hline Lurocalis semitorquatus (Gmelin, 1789) & tuju & & $\mathrm{r}$ & 35,09 & ta \\
\hline Hydropsalis albicollis (Gmelin, 1789) & bacurau & & $\mathrm{r}$ & 7,02 & te \\
\hline Hydropsalis torquata (Gmelin, 1789) & bacurau-tesoura & & $\mathrm{r}$ & 5,26 & te \\
\hline Hydropsalis forcipata (Nitzsch, 1840) & bacurau-tesoura-gigante & $(\mathrm{I}-\mathrm{NT})$ & $\mathrm{r}$ & 3,51 & te \\
\hline \multicolumn{6}{|l|}{ Ordem Apodiformes Peters, 1940} \\
\hline \multicolumn{6}{|l|}{ Família Apodidae Olphe-Galliard, 1887} \\
\hline Streptoprocne zonaris (Shaw, 1796) & taperuçu-de-coleira-branca & & $\mathrm{r}$ & 10,53 & aeri \\
\hline Chaetura cinereiventris Sclater, 1862 & andorinhão-de-sobre-cinzento & & $\mathrm{r}$ & 3,51 & aeri \\
\hline Chaetura meridionalis Hellmayr, 1907 & andorinhão-do-temporal & & $\mathrm{rm}$ & 21,05 & aeri \\
\hline \multicolumn{6}{|l|}{ Família Trochilidae Vigors, 1825} \\
\hline Phaethornis squalidus (Temminck, 1822) & rabo-branco-pequeno & & $\mathrm{r}$ & 19,3 & ta \\
\hline Phaethornis pretrei (Lesson \& Delattre, 1839) & rabo-branco-acanelado & & $\mathrm{r}$ & 15,79 & ta \\
\hline Phaethornis eurynome (Lesson, 1832) & rabo-branco-de-garganta-rajada & & $\mathrm{r}$ & 14,04 & ta \\
\hline Florisuga fusca (Vieillot, 1817) & beija-flor-preto & & $\mathrm{r}$ & 1,75 & ta \\
\hline Colibri serrirostris (Vieillot, 1816) & beija-flor-de-orelha-violeta & & $\mathrm{r}$ & 5,26 & camp \\
\hline Stephanoxis lalandi (Vieillot, 1818) & beija-flor-de-topete & & $\mathrm{r}$ & 14,04 & ta \\
\hline Chlorostilbon lucidus (Shaw, 1812) & besourinho-de-bico-vemelho & & $\mathrm{r}$ & 26,32 & ta \\
\hline Thalurania glaucopis (Gmelin, 1788) & beija-flor-de-fronte-violeta & & $\mathrm{r}$ & 10,53 & ta \\
\hline Hylocharis chrysura (Shaw, 1812) & beija-flor-dourado & & $\mathrm{r}$ & 15,79 & ta \\
\hline Leucochloris albicollis (Vieillot, 1818) & beija-flor-de-papo-branco & & $\mathrm{r}$ & 3,51 & ta \\
\hline Amazilia fimbriata (Gmelin, 1788) & beija-flor-de-garganta-verde & & $\mathrm{r}$ & 1,75 & ta \\
\hline \multirow{2}{*}{\multicolumn{6}{|c|}{$\begin{array}{c}\text { Ordem Trogoniformes A. O. U., } 1886 \\
\text { Família Trogonidae Lesson, } 1828\end{array}$}} \\
\hline & & & & & \\
\hline Trogon surrucura Vieillot, 1817 & surucuá-variado & & $\mathrm{r}$ & 80,7 & ta \\
\hline Trogon rufus Gmelin, 1788 & surucuá-de-barriga-amarela & & $\mathrm{r}$ & 1,75 & ta \\
\hline \multicolumn{6}{|l|}{ Ordem Coraciiformes Forbes, 1844} \\
\hline \multicolumn{6}{|l|}{ Família Alcedinidae Rafinesque, 1815} \\
\hline Megaceryle torquata (Linnaeus, 1766) & martim-pescador-grande & & $\mathrm{r}$ & 24,56 & $\lim$ \\
\hline Chloroceryle amazona (Latham, 1790) & martim-pescador-verde & & $\mathrm{r}$ & 19,3 & $\lim$ \\
\hline Chloroceryle americana (Gmelin, 1788) & martim-pescador-pequeno & & $\mathrm{r}$ & 19,3 & $\lim$ \\
\hline \multicolumn{6}{|l|}{ Família Momotidae Gray, 1840} \\
\hline Baryphthengus ruficapillus (Vieillot, 1818) & juruva-verde & & $\mathrm{r}$ & 85,96 & ta \\
\hline
\end{tabular}

$\mathrm{NT}$ = quase ameaçadas; VU- vulnerável e DD = dados deficientes; Status (St), onde r = residente; rm = residente migratório; vn = visitantes do hemisfério norte. Freqüência de ocorrência $(\mathrm{FO} \%)$; Distribuição no ambiente $(\mathrm{DA})$, onde te $=$ terrícola; ta $=$ tamnícola; co $=$ corticícola; nat $=$ natante; lim $=$ limícola; camp = campícola; aeri $=$ aerícola. Ordenamento taxonômico segue CBRO (2010).

NT = almost endangered; VU- vulnerable e DD = insuficient data; Ocorrence frequency (FO); Habitat distribution (DA), being, te = ground species; ta = branches species; $c o=$ trunk species; nat $=$ waterline species; $\lim =$ adjancent water areas; camp $=$ prairie species; aeri $=$ aerial species Taxonomic order recommended by CBRO (2010). 
Apêndice 1. Continuação...

Appendix 1. Continued..

\begin{tabular}{|c|c|c|c|c|c|}
\hline Taxons & Nome popular & EA (I-E) & ST & FO $(\%)$ & DA \\
\hline \multicolumn{6}{|l|}{ Ordem Galbuliformes Fürbringer, 1888} \\
\hline \multicolumn{6}{|l|}{ Família Bucconidae Horsfield, 1821} \\
\hline Notharchus swainsoni (Gray, 1846) & macuru-de-barriga-castanha & & $\mathrm{r}$ & 8,77 & ta \\
\hline Nonnula rubecula (Spix, 1824) & macuru & & $\mathrm{r}$ & 1,75 & ta \\
\hline \multicolumn{6}{|l|}{ Ordem Piciformes Meyer \& Wolf, 1810} \\
\hline \multicolumn{6}{|l|}{ Família Ramphastidae Vigors, 1825} \\
\hline Selenidera maculirostris (Lichtenstein, 1823) & araçari-poca & & $\mathrm{r}$ & 70,18 & ta \\
\hline Pteroglossus bailloni (Vieillot, 1819) & araçari-banana & $(\mathrm{I}-\mathrm{N})$ & $\mathrm{r}$ & 7,02 & ta \\
\hline Pteroglossus aracari (Linnaeus, 1758) & araçari-de-bico-branco & $(E-V U)$ & $\mathrm{r}$ & 70,18 & ta \\
\hline \multicolumn{6}{|l|}{ Família Picidae Leach, 1820} \\
\hline Picumnus temminckii Lafresnaye, 1845 & pica-pau-anão-de-coleira & & $\mathrm{r}$ & 78,95 & co \\
\hline Picumnus nebulosus Sundevall, 1866 & pica-pau-anão-carijó & & $\mathrm{r}$ & 1,75 & co \\
\hline Melanerpes candidus (Otto, 1796) & birro, pica-pau-branco & & $\mathrm{r}$ & 15,79 & co \\
\hline Melanerpes flavifrons (Vieillot, 1818) & benedito-de-testa-amarela & & $\mathrm{r}$ & 40,35 & co \\
\hline Veniliornis spilogaster (Wagler, 1827) & picapauzinho-verde-carijó & & $\mathrm{r}$ & 54,39 & co \\
\hline Colaptes melanochloros (Gmelin, 1788) & pica-pau-verde-barrado & & $\mathrm{r}$ & 31,58 & co \\
\hline Colaptes campestris (Vieillot, 1818) & pica-pau-do-campo & & $\mathrm{r}$ & 7,02 & camp \\
\hline Celeus flavescens (Gmelin, 1788) & pica-pau-de-cabeça-amarela & & $\mathrm{r}$ & 68,42 & co \\
\hline Dryocopus lineatus (Linnaeus, 1766) & pica-pau-de-banda-branca & & $\mathrm{r}$ & 33,33 & co \\
\hline \multicolumn{6}{|l|}{ Ordem Passeriformes Linné, 1758} \\
\hline \multicolumn{6}{|l|}{ Família Thamnophilidae Swainson, 1824} \\
\hline Hypoedaleus guttatus (Vieillot, 1816) & chocão-carijó & & $\mathrm{r}$ & 31,58 & ta \\
\hline Mackenziaena leachii (Such, 1825) & borralhara-assobiadora & & $\mathrm{r}$ & 10,53 & te \\
\hline Mackenziaena severa (Lichtenstein, 1823) & borralhara & & $\mathrm{r}$ & 21,05 & te \\
\hline Thamnophilus doliatus (Linnaeus, 1764) & choca-barrada & & $\mathrm{r}$ & 7,02 & ta \\
\hline Thamnophilus caerulescens Vieillot, 1816 & choca-da-mata & & $\mathrm{r}$ & 70,18 & ta \\
\hline Dysithamnus mentalis (Temminck, 1823) & choquinha-lisa & & $\mathrm{r}$ & 36,84 & ta \\
\hline Drymophila rubricollis (Bertoni, 1901) & trovoada-de-bertoni & & $\mathrm{r}$ & 1,75 & ta \\
\hline Pyriglena leucoptera (Vieillot, 1818) & papa-taoca-do-sul & & $\mathrm{r}$ & 7,02 & ta \\
\hline \multicolumn{6}{|l|}{ Família Conopophagidae Sclater \& Salvin, 1873} \\
\hline Conopophaga lineata (Wied, 1831) & chupa-dente & & $\mathrm{r}$ & 17,54 & ta \\
\hline \multicolumn{6}{|l|}{ Família Grallariidae Sclater \& Salvin, 1873} \\
\hline Grallaria varia (Boddaert, 1783) & tovacuçu & & $\mathrm{r}$ & 10,53 & te \\
\hline \multicolumn{6}{|l|}{ Família Rhinocryptidae Wetmore, 1930} \\
\hline Eleoscytalopus indigoticus (Wied, 1831) & macuquinho & & $\mathrm{r}$ & 1,75 & ta \\
\hline \multicolumn{6}{|l|}{ Família Formicariidae Gray, 1840} \\
\hline Chamaeza campanisona (Lichtenstein, 1823) & tovaca-campainha & & $\mathrm{r}$ & 8,77 & te \\
\hline \multicolumn{6}{|l|}{ Família Dendrocolaptidae Gray, 1840} \\
\hline Dendrocincla turdina (Lichtenstein, 1820) & arapaçu-liso & & $\mathrm{r}$ & 7,02 & co \\
\hline Sittasomus griseicapillus (Vieillot, 1818) & arapaçu-verde & & $\mathrm{r}$ & 21,05 & co \\
\hline Xiphocolaptes albicollis (Vieillot, 1818) & arapaçu-de-garganta-branca & & $\mathrm{r}$ & 38,6 & co \\
\hline Dendrocolaptes platyrostris Spix, 1825 & arapaçu-grande & & $\mathrm{r}$ & 49,12 & co \\
\hline $\begin{array}{l}\text { Lepidocolaptes falcinellus (Cabanis \& } \\
\text { Heine, 1859) }\end{array}$ & arapaçu-escamado-do-sul & & $\mathrm{r}$ & 12,28 & co \\
\hline \multicolumn{6}{|l|}{ Família Furnariidae Gray, 1840} \\
\hline Furnarius rufus (Gmelin, 1788) & joão-de-barro & & $\mathrm{r}$ & 14,04 & camp \\
\hline Synallaxis ruficapilla Vieillot, 1819 & pichororé & & $\mathrm{r}$ & 14,04 & ta \\
\hline Synallaxis frontalis Pelzeln, 1859 & petrim & & $\mathrm{r}$ & 36,84 & ta \\
\hline Synallaxis spixi Sclater, 1856 & joão-teneném & & $\mathrm{r}$ & 15,79 & ta \\
\hline
\end{tabular}

NT = quase ameaçadas; VU- vulnerável e DD = dados deficientes; Status (St), onde $\mathrm{r}=$ residente; $\mathrm{rm}$ = residente migratório; vn = visitantes do hemisfério norte. Frequiência de ocorrência (FO\%); Distribuição no ambiente $(\mathrm{DA})$, onde te = terrícola; ta = tamnícola; co = corticícola; nat = natante; lim = limícola; camp = campícola; aeri = aerícola. Ordenamento taxonômico segue CBRO (2010).

$\mathrm{NT}=$ almost endangered; VU- vulnerable e DD = insuficient data; Ocorrence frequency (FO); Habitat distribution $(\mathrm{DA})$, being, te $=$ ground species; ta $=$ branches species; co = trunk species; nat = waterline species; lim = adjancent water areas; camp = prairie species; aeri = aerial species. Taxonomic order recommended by CBRO (2010). 
Apêndice 1. Continuação...

Appendix 1. Continued...

\begin{tabular}{|c|c|c|c|c|c|}
\hline Taxons & Nome popular & EA (I-E) & ST & FO $(\%)$ & DA \\
\hline Cranioleuca obsoleta (Reichenbach, 1853) & arredio-oliváceo & & $\mathrm{r}$ & 14,04 & ta \\
\hline Certhiaxis cinnamomeus (Gmelin, 1788) & curutié & & $\mathrm{r}$ & 5,26 & ta \\
\hline Syndactyla rufosuperciliata (Lafresnaye, 1832) & trepador-quiete & & $\mathrm{r}$ & 5,26 & ta \\
\hline Philydor rufum (Vieillot, 1818) & limpa-folha-de-testa-baia & & $\mathrm{r}$ & 1,75 & co \\
\hline Automolus leucophthalmus (Wied, 1821) & barranqueiro-de-olho-branco & & $\mathrm{r}$ & 82,46 & ta \\
\hline Heliobletus contaminatus Berlepsch, 1885 & trepadorzinho & & $\mathrm{r}$ & 12,28 & ta \\
\hline Xenops rutilans Temminck, 1821 & bico-virado-carijó & & $\mathrm{r}$ & 38,6 & ta \\
\hline \multicolumn{6}{|l|}{$\begin{array}{l}\text { Família Rhynchocyclidae Tello, Moyle, Marchese \& } \\
\text { Cracraft } 2009\end{array}$} \\
\hline Tolmomyias sulphurescens (Spix, 1825) & bico-chato-de-orelha-preta & & $\mathrm{r}$ & 45,61 & ta \\
\hline Poecilotriccus plumbeiceps (Lafresnaye, 1846) & tororó & & $\mathrm{r}$ & 5,26 & ta \\
\hline Leptopogon amaurocephalus Tschudi, 1846 & cabeçudo & & $\mathrm{r}$ & 75,44 & ta \\
\hline Myiornis auricularis (Vieillot, 1818) & miudinho & & $\mathrm{r}$ & 22,81 & ta \\
\hline Mionectes rufiventris Cabanis, 1846 & abre-asa-de-cabeça-cinza & & $\mathrm{r}$ & 12,28 & ta \\
\hline Corythopis delalandi (Lesson, 1830) & estalador & & $\mathrm{r}$ & 38,6 & ta \\
\hline Phylloscartes eximius (Temminck, 1822) & barbudinho & $(\mathrm{I}-\mathrm{NT})$ & $\mathrm{r}$ & 5,26 & ta \\
\hline Phylloscartes ventralis (Temminck, 1824) & borboletinha-do-mato & & $\mathrm{r}$ & 28,07 & ta \\
\hline Phylloscartes sylviolus (Cabanis \& Heine, 1859) & maria-pequena & & $\mathrm{r}$ & 1,75 & ta \\
\hline \multicolumn{6}{|l|}{ Família Tyrannidae Vigors, 1825} \\
\hline Phyllomyias virescens (Temminck, 1824) & piolhinho-verdoso & & $\mathrm{r}$ & 1,75 & ta \\
\hline Myiopagis caniceps (Swainson, 1835) & guaracava-cinzenta & & $\mathrm{r}$ & 19,3 & ta \\
\hline Myiopagis viridicata (Vieillot, 1817) & guaracava-de-crista-alaranjada & & $\mathrm{r}$ & 38,6 & ta \\
\hline Elaenia flavogaster (Thunberg, 1822) & guaracava-de-barriga-amarela & & $\mathrm{r}$ & 35,09 & ta \\
\hline Elaenia parvirostris Pelzeln, 1868 & guaracava-de-crista-branca & & $\mathrm{r}$ & 7,02 & ta \\
\hline Elaenia obscura (d’Orbgny \& Lafresnaye, 1837) & guaracava-de-topete-vermelho & & $\mathrm{r}$ & 5,26 & ta \\
\hline Camptostoma obsoletum (Temminck, 1824) & risadinha & & $\mathrm{r}$ & 36,84 & ta \\
\hline Serpophaga nigricans (Vieillot, 1817) & joão-pobre & & $\mathrm{r}$ & 1,75 & ta \\
\hline Serpophaga subcristata (Vieillot, 1817) & alegrinho & & $\mathrm{r}$ & 15,79 & ta \\
\hline Phaeomyias murina (Spix, 1825) & bagageiro & & $\mathrm{r}$ & 1,75 & ta \\
\hline Capsiempis flaveola (Lichtenstein, 1823) & marianinha-amarela & & $\mathrm{r}$ & 73,68 & ta \\
\hline Euscarthmus meloryphus Wied, 1831 & barulhento & & $\mathrm{r}$ & 50,88 & ta \\
\hline Sublegatus modestus (Wied, 1831) & guaracava-modesta & & $\mathrm{r}$ & 1,75 & ta \\
\hline Piprites chloris (Temminck, 1822) & papinho-amarelo & & $\mathrm{r}$ & 1,75 & ta \\
\hline Myiophobus fasciatus (Statius Muller, 1776) & filipe & & $\mathrm{r}$ & 21,05 & ta \\
\hline Hirundinea ferruginea (Gmelin, 1788) & gibão-de-couro & & $\mathrm{r}$ & 7,02 & ta \\
\hline Lathrotriccus euleri (Cabanis, 1868) & enferrujado & & $\mathrm{r}$ & 42,11 & ta \\
\hline Cnemotriccus fuscatus (Wied, 1831) & guaracavuçu & & $\mathrm{r}$ & 40,35 & ta \\
\hline Contopus cinereus (Spix, 1825) & papa-moscas-cinzento & & $\mathrm{r}$ & 24,56 & ta \\
\hline Pyrocephalus rubinus (Boddaert, 1783) & príncipe & & $\mathrm{rm}$ & 1,75 & camp \\
\hline Knipolegus cyanirostris (Vieillot, 1818) & maria-preta-de-bico-azulado & & $\mathrm{r}$ & 29,82 & ta \\
\hline Satrapa icterophrys (Vieillot, 1818) & suiriri-pequeno & & $\mathrm{r}$ & 1,75 & ta \\
\hline Muscipipra vetula (Lichtenstein, 1823) & tesoura-cinzenta & $(\mathrm{I}-\mathrm{NT})$ & $\mathrm{r}$ & 10,53 & ta \\
\hline Colonia colonus (Vieillot, 1818) & viuvinha & & $\mathrm{r}$ & 10,53 & ta \\
\hline Machetornis rixosa (Vieillot, 1819) & suiriri-cavaleiro & & $\mathrm{r}$ & 22,81 & camp \\
\hline Legatus leucophaius (Vieillot, 1818) & bem-te-vi-pirata & & $\mathrm{r}$ & 19,3 & ta \\
\hline Myiozetetes similis (Spix, 1825) & bentevizinho-de-penacho-vermelho & & $\mathrm{r}$ & 66,67 & ta \\
\hline Pitangus sulphuratus (Linnaeus, 1766) & bem-te-vi & & $\mathrm{r}$ & 89,47 & ta \\
\hline Conopias trivirgatus (Wied, 1831) & bem-te-vi-pequeno & & $\mathrm{r}$ & 17,54 & ta \\
\hline Myiodynastes maculatus (Statius Muller, 1776) & bem-te-vi-rajado & & $\mathrm{r}$ & 43,86 & ta \\
\hline
\end{tabular}

NT = quase ameaçadas; VU- vulnerável e DD = dados deficientes; Status (St), onde r = residente; $r m$ = residente migratório; vn = visitantes do hemisfério norte. Freqüência de ocorrência $(\mathrm{FO} \%)$; Distribuição no ambiente $(\mathrm{DA})$, onde te = terrícola; ta = tamnícola; co = corticícola; nat = natante; lim = limícola; camp = campícola; aeri = aerícola. Ordenamento taxonômico segue CBRO (2010).

$\mathrm{NT}=$ almost endangered; VU- vulnerable e DD = insuficient data; Ocorrence frequency (FO); Habitat distribution $(\mathrm{DA})$, being, te = ground species; ta = branches species; $c o=$ trunk species; nat $=$ waterline species; $\lim =$ adjancent water areas; camp $=$ prairie species; aeri $=$ aerial species. Taxonomic order recommended by CBRO (2010). 
Apêndice 1. Continuação...

Appendix 1. Continued...

\begin{tabular}{|c|c|c|c|c|c|}
\hline Taxons & Nome popular & EA (I-E) & ST & FO $(\%)$ & DA \\
\hline Megarynchus pitangua (Linnaeus, 1766) & neinei & & $\mathrm{r}$ & 92,98 & ta \\
\hline Empidonomus varius (Vieillot, 1818) & peitica & & $\mathrm{r}$ & 7,02 & ta \\
\hline Tyrannus melancholicus Vieillot, 1819 & suiriri & & $\mathrm{r}$ & 31,58 & ta \\
\hline Tyrannus savana Vieillot, 1808 & tesourinha & & $\mathrm{rm}$ & 24,56 & camp \\
\hline Sirystes sibilator (Vieillot, 1818) & gritador & & r & 61,4 & ta \\
\hline Myiarchus swainsoni Cabanis \& Heine, 1859 & irré & & $\mathrm{r}$ & 8,77 & ta \\
\hline Myiarchus ferox (Gmelin, 1789) & maria-cavaleira & & $\mathrm{r}$ & 12,28 & ta \\
\hline Myiarchus tyrannulus (Statius Muller, 1776) & $\begin{array}{l}\text { maria-cavaleira-de- } \\
\text { rabo-enferrujado }\end{array}$ & & $\mathrm{r}$ & 1,75 & ta \\
\hline Attila phoenicurus Pelzeln, 1868 & capitão-castanho & & $\mathrm{r}$ & 5,26 & ta \\
\hline \multicolumn{6}{|l|}{ Família Cotingidae Bonaparte, 1849} \\
\hline Phibalura flavirostris Vieillot, 1816 & tesourinha-da-mata & $(\mathrm{I}-\mathrm{NT})$ & $\mathrm{r}$ & 3,51 & ta \\
\hline Procnias nudicollis (Vieillot, 1817) & araponga & $(\mathrm{I}-\mathrm{NT})$ & $\mathrm{r}$ & 8,77 & ta \\
\hline \multicolumn{6}{|l|}{ Família Pipridae Rafinesque, 1815} \\
\hline Neopelma pallescens (Lafresnaye, 1853) & fruxu-do-cerradão & & $\mathrm{r}$ & 1,75 & ta \\
\hline Chiroxiphia caudata (Shaw \& Nodder, 1793) & tangará & & $\mathrm{r}$ & 29,82 & ta \\
\hline Pipra fasciicauda Hellmayr, 1906 & uirapuru-laranja & & $\mathrm{r}$ & 78,95 & ta \\
\hline \multicolumn{6}{|l|}{ Família Tityridae Gray, 1840} \\
\hline Oxyruncus cristatus Swainson, 1821 & araponga-do-horto & & $\mathrm{r}$ & 14,04 & ta \\
\hline Schiffornis virescens (Lafresnaye, 1838) & flautim & & $\mathrm{r}$ & 10,53 & ta \\
\hline Laniisoma elegans (Thunberg, 1823) & chibante & $\begin{array}{l}\text { (I - VU; } \\
\text { E - DD) }\end{array}$ & r & 1,75 & ta \\
\hline Tityra inquisitor (Lichtenstein, 1823) & anambé-branco-de-bochecha-parda & & $\mathrm{r}$ & 19,3 & ta \\
\hline Tityra cayana (Linnaeus, 1766) & anambé-branco-de-rabo-preto & & $\mathrm{r}$ & 70,18 & ta \\
\hline Pachyramphus viridis (Vieillot, 1816) & caneleiro-verde & & $\mathrm{r}$ & 5,26 & ta \\
\hline Pachyramphus castaneus (Jardine \& Selby, 1827) & caneleiro & & $\mathrm{r}$ & 8,77 & ta \\
\hline Pachyramphus polychopterus (Vieillot, 1818) & caneleiro-preto & & $\mathrm{r}$ & 5,26 & ta \\
\hline Pachyramphus validus (Lichtenstein, 1823) & caneleiro-de-chapéu-preto & & $\mathrm{r}$ & 38,6 & ta \\
\hline \multicolumn{6}{|l|}{ Família Vireonidae Swainson, 1837} \\
\hline Cyclarhis gujanensis (Gmelin, 1789) & pitiguari & & $\mathrm{r}$ & 71,93 & ta \\
\hline Vireo olivaceus (Linnaeus, 1766) & juruviara & & $\mathrm{r}$ & 28,07 & ta \\
\hline Hylophilus amaurocephalus (Nordmann, 1835) & vite-vite-de-olho-cinza & $(\mathrm{E}-\mathrm{DD})$ & $\mathrm{r}$ & 1,75 & ta \\
\hline \multicolumn{6}{|l|}{ Família Corvidae Leach, 1820} \\
\hline Cyanocorax chrysops (Vieillot, 1818) & gralha-picaça & & $\mathrm{r}$ & 75,44 & ta \\
\hline \multicolumn{6}{|l|}{ Família Hirundinidae Rafinesque, 1815} \\
\hline Pygochelidon cyanoleuca (Vieillot, 1817) & andorinha-pequena-de-casa & & $\mathrm{rm}$ & 31,58 & camp \\
\hline Stelgidopteryx ruficollis (Vieillot, 1817) & andorinha-serradora & & $\mathrm{rm}$ & 24,56 & camp \\
\hline Progne tapera (Vieillot, 1817) & andorinha-do-campo & & $\mathrm{rm}$ & 1,75 & camp \\
\hline Progne chalybea (Gmelin, 1789) & andorinha-doméstica-grande & & $\mathrm{rm}$ & 21,05 & camp \\
\hline Tachycineta albiventer (Boddaert, 1783) & andorinha-do-rio & & $\mathrm{r}$ & 36,84 & $\lim$ \\
\hline Tachycineta leucorrhoa (Vieillot, 1817) & andorinha-de-sobre-branco & & $\mathrm{r}$ & 19,3 & camp \\
\hline Hirundo rustica Linnaeus, 1758 & andorinha-de-bando & & vn & 3,51 & camp \\
\hline \multicolumn{6}{|l|}{ Família Troglodytidae Swainson, 1831} \\
\hline Troglodytes musculus Naumann, 1823 & corruíra & & $\mathrm{r}$ & 28,07 & camp \\
\hline \multicolumn{6}{|l|}{ Família Donacobiidae Aleixo \& Pacheco, 2006} \\
\hline Donacobius atricapilla (Linnaeus, 1766) & japacanim & & $\mathrm{r}$ & 15,79 & $\lim$ \\
\hline \multicolumn{6}{|l|}{ Família Turdidae Rafinesque, 1815} \\
\hline Turdus rufiventris Vieillot, 1818 & sabiá-laranjeira & & $\mathrm{r}$ & 47,37 & ta \\
\hline Turdus leucomelas Vieillot, 1818 & sabiá-barranco & & $\mathrm{r}$ & 85,96 & ta \\
\hline Turdus amaurochalinus Cabanis, 1850 & sabiá-poca & & $\mathrm{rm}$ & 59,65 & ta \\
\hline
\end{tabular}

NT = quase ameaçadas; VU- vulnerável e DD = dados deficientes; Status $(\mathrm{St})$, onde $\mathrm{r}=$ residente; $\mathrm{rm}=$ residente migratório; vn $=$ visitantes do hemisfério norte. Freqüência de ocorrência (FO\%); Distribuição no ambiente $(\mathrm{DA})$, onde te = terrícola; ta = tamnícola; co = corticícola; nat = natante; lim = limícola; camp = campícola; aeri $=$ aerícola. Ordenamento taxonômico segue CBRO (2010).

$\mathrm{NT}=$ almost endangered; VU- vulnerable e DD = insuficient data; Ocorrence frequency $(\mathrm{FO})$; Habitat distribution $(\mathrm{DA})$, being, te $=$ ground species; ta = branches species; $\mathrm{co}=$ trunk species; nat $=$ waterline species; $\mathrm{lim}=$ adjancent water areas; camp = prairie species; aeri = aerial species. Taxonomic order recommended by CBRO (2010). 
Apêndice 1. Continuação...

Appendix 1. Continued...

\begin{tabular}{|c|c|c|c|c|c|}
\hline Taxons & Nome popular & EA (I-E) & ST & FO $(\%)$ & DA \\
\hline Turdus subalaris (Seebohm, 1887) & sabiá-ferreiro & & $\mathrm{rm}$ & 22,81 & ta \\
\hline Turdus albicollis Vieillot, 1818 & sabiá-coleira & & $\mathrm{r}$ & 42,11 & ta \\
\hline \multicolumn{6}{|l|}{ Família Mimidae Bonaparte, 1853} \\
\hline Mimus saturninus (Lichtenstein, 1823) & sabiá-do-campo & & $\mathrm{r}$ & 3,51 & camp \\
\hline \multicolumn{6}{|l|}{ Família Coerebidae d'Orbigny \& Lafresnaye, 1838} \\
\hline Coereba flaveola (Linnaeus, 1758) & cambacica & & $\mathrm{r}$ & 5,26 & ta \\
\hline \multicolumn{6}{|l|}{ Família Thraupidae Cabanis, 1847} \\
\hline Saltator similis d'Orbigny \& Lafresnaye, 1837 & trinca-ferro-verdadeiro & & $\mathrm{r}$ & 64,91 & ta \\
\hline Nemosia pileata (Boddaert, 1783) & saíra-de-chapéu-preto & & $\mathrm{r}$ & 8,77 & ta \\
\hline Pyrrhocoma ruficeps (Strickland, 1844) & cabecinha-castanha & & $\mathrm{r}$ & 24,56 & ta \\
\hline Tachyphonus coronatus (Vieillot, 1822) & tiê-preto & & $\mathrm{r}$ & 33,33 & ta \\
\hline Lanio cucullatus (Statius Muller, 1776) & tico-tico-rei & & $\mathrm{r}$ & 56,14 & ta \\
\hline Lanio melanops (Vieillot, 1818) & tiê-de-topete & & $\mathrm{r}$ & 49,12 & ta \\
\hline Tangara seledon (Statius Muller, 1776) & saíra-sete-cores & & $\mathrm{r}$ & 8,77 & ta \\
\hline Tangara sayaca (Linnaeus, 1766) & sanhaçu-cinzento & & $\mathrm{r}$ & 47,37 & ta \\
\hline Tangara peruviana (Desmarest, 1806) & saíra-sapucaia & & $\mathrm{r}$ & 5,26 & ta \\
\hline Cissopis leverianus (Gmelin, 1788) & tietinga & & $\mathrm{r}$ & 49,12 & ta \\
\hline Pipraeidea melanonota (Vieillot, 1819) & saíra-viúva & & $\mathrm{r}$ & 29,82 & ta \\
\hline Tersina viridis (Illiger, 1811) & saí-andorinha & & $\mathrm{r}$ & 15,79 & ta \\
\hline Dacnis cayana (Linnaeus, 1766) & saí-azul & & $\mathrm{r}$ & 50,88 & ta \\
\hline Hemithraupis guira (Linnaeus, 1766) & saíra-de-papo-preto & & $\mathrm{r}$ & 82,46 & ta \\
\hline Conirostrum speciosum (Temminck, 1824) & figuinha-de-rabo-castanho & & $\mathrm{r}$ & 75,44 & ta \\
\hline \multicolumn{6}{|l|}{ Família Emberizidae Vigors, 1825} \\
\hline Zonotrichia capensis (Statius Muller, 1776) & tico-tico & & $\mathrm{r}$ & 24,56 & camp \\
\hline Haplospiza unicolor Cabanis, 1851 & cigarra-bambu & & $\mathrm{r}$ & 7,02 & ta \\
\hline Sicalis flaveola (Linnaeus, 1766) & canário-da-terra-verdadeiro & & $\mathrm{r}$ & 1,75 & camp \\
\hline Volatinia jacarina (Linnaeus, 1766) & tiziu & & $\mathrm{r}$ & 17,54 & camp \\
\hline Sporophila caerulescens (Vieillot, 1823) & coleirinho & & $\mathrm{r}$ & 29,82 & camp \\
\hline \multicolumn{6}{|l|}{ Família Cardinalidae Ridgway, 1901} \\
\hline Habia rubica (Vieillot, 1817) & tiê-do-mato-grosso & & $\mathrm{r}$ & 21,05 & ta \\
\hline Cyanoloxia moesta (Hartlaub, 1853) & negrinho-do-mato & & $\mathrm{r}$ & 1,75 & ta \\
\hline $\begin{array}{l}\text { Cyanoloxia glaucocaerulea (d'Orbigny \& } \\
\text { Lafresnaye, 1837) }\end{array}$ & azulinho & $(\mathrm{E}-\mathrm{NT})$ & $\mathrm{r}$ & 5,26 & ta \\
\hline \multicolumn{6}{|l|}{$\begin{array}{l}\text { Família Parulidae Wetmore, Friedmann, Lincoln, } \\
\text { Miller, Peters, van Rossem, Van Tyne \& Zimmer } 1947\end{array}$} \\
\hline Parula pitiayumi (Vieillot, 1817) & mariquita & & $\mathrm{r}$ & 59,65 & ta \\
\hline Geothlypis aequinoctialis (Gmelin, 1789) & pia-cobra & & $\mathrm{r}$ & 17,54 & ta \\
\hline Basileuterus culicivorus (Deppe, 1830) & pula-pula & & $\mathrm{r}$ & 91,23 & ta \\
\hline Basileuterus leucoblepharus (Vieillot, 1817) & pula-pula-assobiador & & $\mathrm{r}$ & 1,75 & ta \\
\hline \multicolumn{6}{|l|}{ Família Icteridae Vigors, 1825} \\
\hline Cacicus haemorrhous (Linnaeus, 1766) & guaxe & & $\mathrm{r}$ & 42,11 & ta \\
\hline Icterus cayanensis (Linnaeus, 1766) & encontro & & $\mathrm{r}$ & 14,04 & ta \\
\hline Pseudoleistes guirahuro (Vieillot, 1819) & chopim-do-brejo & & $\mathrm{r}$ & 1,75 & camp \\
\hline Molothrus oryzivorus (Gmelin, 1788) & iraúna-grande & & $\mathrm{r}$ & 3,51 & camp \\
\hline Molothrus bonariensis (Gmelin, 1789) & vira-bosta & & $\mathrm{r}$ & 8,77 & camp \\
\hline \multicolumn{6}{|l|}{ Família Fringillidae Leach, 1820} \\
\hline Sporagras magellanica (Vieillot, 1805) & pintassilgo & & $\mathrm{r}$ & 10,53 & camp \\
\hline Euphonia chlorotica (Linnaeus, 1766) & fim-fim & & $\mathrm{r}$ & 42,11 & ta \\
\hline Euphonia violacea (Linnaeus, 1758) & gaturamo-verdadeiro & & $\mathrm{r}$ & 17,54 & ta \\
\hline Euphonia pectoralis (Latham, 1801) & ferro-velho & & $\mathrm{r}$ & 26,32 & ta \\
\hline
\end{tabular}

NT = quase ameaçadas; VU- vulnerável e DD = dados deficientes; Status (St), onde r = residente; rm = residente migratório; vn = visitantes do hemisfério norte. Frequiência de ocorrência $(\mathrm{FO} \%)$; Distribuição no ambiente $(\mathrm{DA})$, onde te = terrícola; ta = tamnícola; co = corticícola; nat = natante; lim = limícola; camp = campícola; aeri $=$ aerícola. Ordenamento taxonômico segue CBRO (2010).

$\mathrm{NT}=$ almost endangered; VU- vulnerable e DD = insuficient data; Ocorrence frequency (FO); Habitat distribution (DA), being, te = ground species; ta = branches species; $\mathrm{co}=$ trunk species; nat $=$ waterline species; $\lim =$ adjancent water areas; camp = prairie species; aeri $=$ aerial species. Taxonomic order recommended by CBRO (2010). 